\title{
New nuclear structure data after fission: The g.s. of ${ }^{136} \mathrm{Sb}$
}

\author{
Radomira Lozeva ${ }^{1,2, *}$, Xiuquan $\mathrm{Li}^{1}$, Aurelien Blanc ${ }^{3}$, Jean-Michel Daugas ${ }^{4}$, François \\ Didierjean $^{2}$, Gilbert Duchêne ${ }^{2}$, Ulli Köster ${ }^{3}$, Teresa Kurtukian-Nieto ${ }^{5}$, François Le Blanc ${ }^{2}$, \\ Paolo Mutti ${ }^{3}$, Mourad Ramdhane ${ }^{6}$, Torsten Soldner ${ }^{3}$, and Waldemar Urban $^{7}$ \\ ${ }^{1}$ CSNSM, CNRS/IN2P3, Université Paris-Sud, 91405 Orsay Campus, Cedex, France \\ ${ }^{2}$ IPHC, CNRS/IN2P3, Université de Strasbourg, 67037 Strasbourg, Cedex 2, France \\ ${ }^{3}$ ILL, 38042 Grenoble, Cedex 9, France \\ ${ }^{4}$ CEA/DAM, 91297 Arpajon, Cedex, France \\ ${ }^{5} \mathrm{CENBG}, \mathrm{CNRS} / \mathrm{IN} 2 \mathrm{P} 3$, Université de Bordeaux, 33170 Gradignan, Cedex, France \\ ${ }^{6}$ LPSC, CNRS/IN2P3, Université de Grenoble Alpes, 38026 Grenoble, Cedex, France \\ ${ }^{7}$ Faculty of Physics, University of Warsaw, 02093 Warszawa, Poland
}

\begin{abstract}
Nuclei in the neutron-rich region beyond ${ }^{132} \mathrm{Sn}$ have been produced recently by various experiments using fission. Using isomer and $\beta$-decay studies nuclear structure data has been collected on the orbital evolution and collectivity in the region with both the increase of proton and neutron numbers. Examples on particular questions related to the g.s. of the $A=136$ odd-odd ${ }^{136} \mathrm{Sb}$ nucleus and its heavier neighbours are given in the scope of expectations by shell-model theory.
\end{abstract}

\section{Introduction}

Exotic nuclei beyond the ${ }^{132} \mathrm{Sn}$ double-shell closure are influenced by both the $\mathrm{Sn}$ superfluidity and the evolving collectivity only few nucleons away. Toward even more neutron-rich nuclei, especially at intermediate mass number, interplay between singleparticle and collective particle-hole excitations starts to compete. In some cases with the extreme addition of neutrons also other effects as the formation of neutron skin [1], stabilization as sub-shell gaps [2], and/or orbital crossings [3] may be expected. The knowledge of nuclear ingredients is especially interesting beyond ${ }^{132} \mathrm{Sn}$ and little is known on how the excitation modes develop with the addition of both protons and neutrons [4-7]. Analogy to the nuclei beyond ${ }^{208} \mathrm{~Pb}$ [8] could be used close to the doubly-shell core, based on the dominant role of the pairing correlations in its vicinity. Little data is available for more than two-proton, four neutron systems [9] and it is unclear whether other components mix in the configuration of states preferentially when neutron rather than proton pair is added into the system. It is a challenge for the today's realistic shell model calculations, that work well for the species around the doubly-closed core, to constrain the effective interaction between the nucleons for such very exotic region with extreme $N$, thus any

\footnotetext{
${ }^{*}$ Corresponding author: radomira.lozeva@,csnsm.in2p3.fr
} 
experimental data is crucial input to theory to allow a global description and valuable theoretical predictions.

\section{Motivation}

Several nuclei in the region draw attention due to various inconsistencies beyond $N=82$ as the fast $B(E 2)$ drop for ${ }^{136} \mathrm{Te}$ with respect to ${ }^{134} \mathrm{Sn},{ }^{136} \mathrm{Sn}[10,11]$. This was suggested to appear due to the excess of neutrons and strong neutron-proton exchange asymmetry, leading to dramatic drop in the $E\left(2 I^{+}\right)$state in ${ }^{138} \mathrm{Te}$ (and ${ }^{140} \mathrm{Xe}$ ) [12]. The first excited $5 / 2^{+}$ state in ${ }^{135} \mathrm{Sb}$ was found to be drastically lower, instead of increasing in energy as for ${ }^{133} \mathrm{Sb}$ $[13,14]$. It was expected that the repulsion between $v f_{7 / 2}$ and $\pi d_{5 / 2}$ orbitals appears stronger due to a reduced pairing with respect e.g. of the nearly degenerate $\pi d_{5 / 2}$ and $\pi g_{7 / 2}$ single particle orbitals, found to cross at an extreme $N=89$ for ${ }^{140} \mathrm{Sb}$ [15]. Orbital evolution between $N=82$ and $N=90$ is still unknown and is given emphasis in recent studies. For example, the ground state spin of ${ }^{136} \mathrm{Sb}$ is unfixed with several possibilities suggested, ranging from $\left(0^{-}, 1^{-}\right.$and $\left.2^{-}\right)$depending on the theoretical interpretation on the very few existing experimental results $[8,16]$. In particular, in an earlier $\beta$-decay experiment a good agreement was achieved with spin/parity of $\left(1^{-}\right)$with $\pi g_{7 / 2} v f_{7 / 2}{ }^{3}$ configuration, while $\left(0^{-}\right)$was ruled out due to the relatively strong first forbidden unique $\beta$ to the $0^{+}$of ${ }^{136} \mathrm{Te}$ but also to unobserved branches. According to [17], the (2) state should be very low in energy and possibly a g.s., as the assumption for spin/parity of $\left(1^{-}\right)$for the g.s. does not allow to theoretically reproduce the experimental spectra of both ${ }^{134,135} \mathrm{Sb}$. We have initiated a new $\beta$-decay study to clarify this question linking to the heavier Sb. For example we have proposed (3-) spin assignment to the g.s. of ${ }^{138} \mathrm{Sb}[18]$ due to a strong $\beta$-decay branch to the $\left(2^{+}\right)$states in the ${ }^{138} \mathrm{Te}$ daughter nucleus build on the $\pi g_{7 / 2} v f_{7 / 2}{ }^{5}$ configuration, in variance to the theoretical $\left(4^{-}\right)[17,19]$. The $\pi g_{7 / 2}-\pi d_{5 / 2}$ orbital inversion we have proposed in [15] together with the observation of the first $2^{+}, 4^{+}$states in ${ }^{140} \mathrm{Te}$ lead us to most probable $\left(3^{-}\right)$ assignment build on the same configuration for the g.s. of ${ }^{140} \mathrm{Sb}[20]$.

\section{Experimental studies}

\subsection{Fission to populate the ${ }^{132} \mathrm{Sn}$ region}

Recently, we have approached the region of nuclei in several measurements following fission of ${ }^{238} \mathrm{U}$ on ${ }^{9} \mathrm{Be}$ target at high energies of $350 \mathrm{MeV} / \mathrm{n}$, within the EURICA project [21-22], thermal $n$-induced fission on ${ }^{235} \mathrm{U} /{ }^{241} \mathrm{Pu}$ targets using prompt-decay spectroscopy within the EXILL/FATIMA campaigns [23,24], as well as in $\beta$-decay spectroscopy at Lohengrin. These complementary studies on several nuclei in the region show coherent picture and possible interpretation of the new data beyond ${ }^{132} \mathrm{Sn}$. Hereafter, we describe first outcomes for the $A=136$ nuclei performed at the ILL.

\section{2 $\beta$-decay fission products at Lohengrin}

Experimental study was performed at the Lohengrin spectrometer on $A=136$ fission fragments produced in ${ }^{235} \mathrm{U}\left(\mathrm{n}_{\mathrm{th}}, \mathrm{f}\right)$ reaction and transported to the focal plane behind a focusing magnet using the technique described in [25]. An experimental setup was built using the $\beta$-decay station of plastic detectors in $4 \pi$ geometry from the LOENIE $\beta$-delayed neutron detector [26]. The box was placed around a vacuum chamber that supported a movable tape, used to evacuate the radioactivity from the implanted ions. The duty cycle 
was adapted to the $t_{1 / 2}$ of the isotopes of interest e.g. the $A=136 \mathrm{Sb}$ ions. Emitted $\gamma$-rays for selected $\beta$-decay event were detected by two Clover detectors (with Anti-Compton (AC) shields) and one standard coaxial HpGe detector in close geometry (see Fig. 1). In addition, $\mathrm{LaBr}_{3}(\mathrm{Ce})$ detectors were added to the $\gamma$-detection to scan lifetimes of the populated states in the sub-ns time range. Further experimental details can be found elsewhere [27].
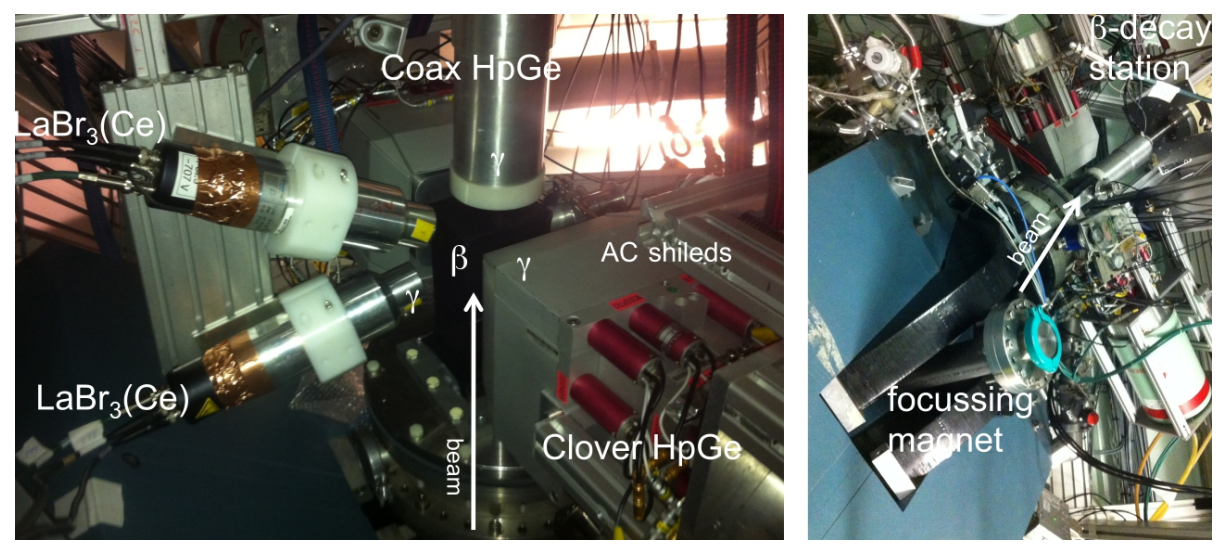

Fig. 1. Experimental setup used at Lohengrin combining $\gamma$-ray detectors and $\beta$-decay station.

\section{Preliminary results}

The experimental data on $A=136$ was collected for several charge states $(Q=21,25,30)$ in order to obtain good ratio between $A=136$ nuclei of interest and contaminants with $A / Q$ accepted by the system. The resulting spectrum for the best peak/contaminant selection corresponding to $Q=21$ is shown in Fig. 2. $\gamma$-rays following the $\beta$-disintegration of the mother nucleus ${ }^{136} \mathrm{Sb}\left(t_{1 / 2}\right.$ of $0.923(14) \mathrm{s}$ [28] $)$ to ${ }^{136} \mathrm{Xe}\left(t_{1 / 2}\right.$ of $2.165 \times 10^{21} \mathrm{y}$ [28] $)$ are identified. The tape duty cycle was optimised for ${ }^{136} \mathrm{Sb}$ to be: $5 \mathrm{~s}$ beam open, $5 \mathrm{~s}$ beam closed and $2 \mathrm{~s}$ tape movement.

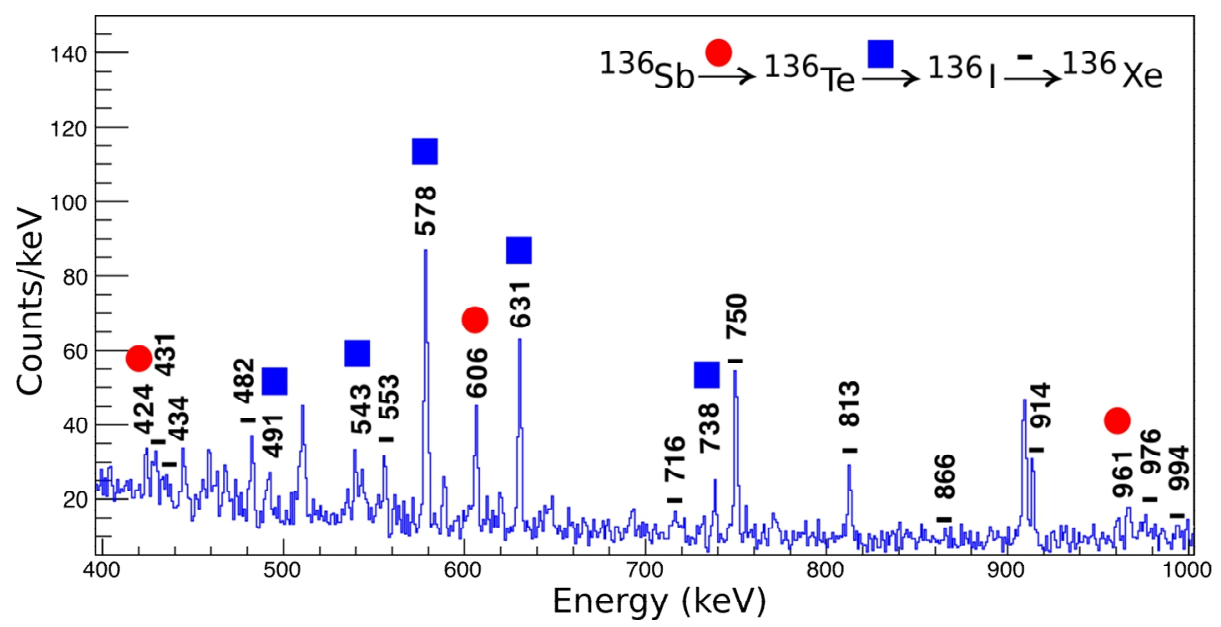

Fig. 2. Experimental $\gamma$-ray spectrum with the transitions in ${ }^{136} \mathrm{Te},{ }^{136} \mathrm{I}$ and ${ }^{136} \mathrm{Xe}$ populated after $\beta$ decay of ${ }^{136} \mathrm{Sb}$. 


\subsection{Identification}

After proper energy and efficiency calibration all observed $\gamma$-rays and their intensities were attributed to the different isobars based on their time behaviour (see Fig. 3). Despite the optimized time cycle, the $\gamma$-rays belonging to the ${ }^{136} \mathrm{Sb} \rightarrow{ }^{136} \mathrm{Te}$ decay were weaker than the ${ }^{136} \mathrm{Te} \rightarrow{ }^{136} \mathrm{I}$ decay. This is due to both, the lower fission yield of ${ }^{136} \mathrm{Sb}$ and the stronger g.s. to g.s. transition in the decay of the latter. We note that no (e.g. long-lived) isomers in these nuclei contribute to the observed transitions. This is not the case for ${ }^{136} \mathrm{I} \rightarrow{ }^{136} \mathrm{Xe}$ decay, where we have detected $\gamma$-rays from the $\left(6^{-}\right) 46.9(10) \mathrm{s}$, state as identified in $[28,29]$. The $\mathrm{P}_{\mathrm{n}}$ branch $\left(16.3 \%\right.$ [28]) for ${ }^{136} \mathrm{Sb}$ was taken care of the analysis [27].

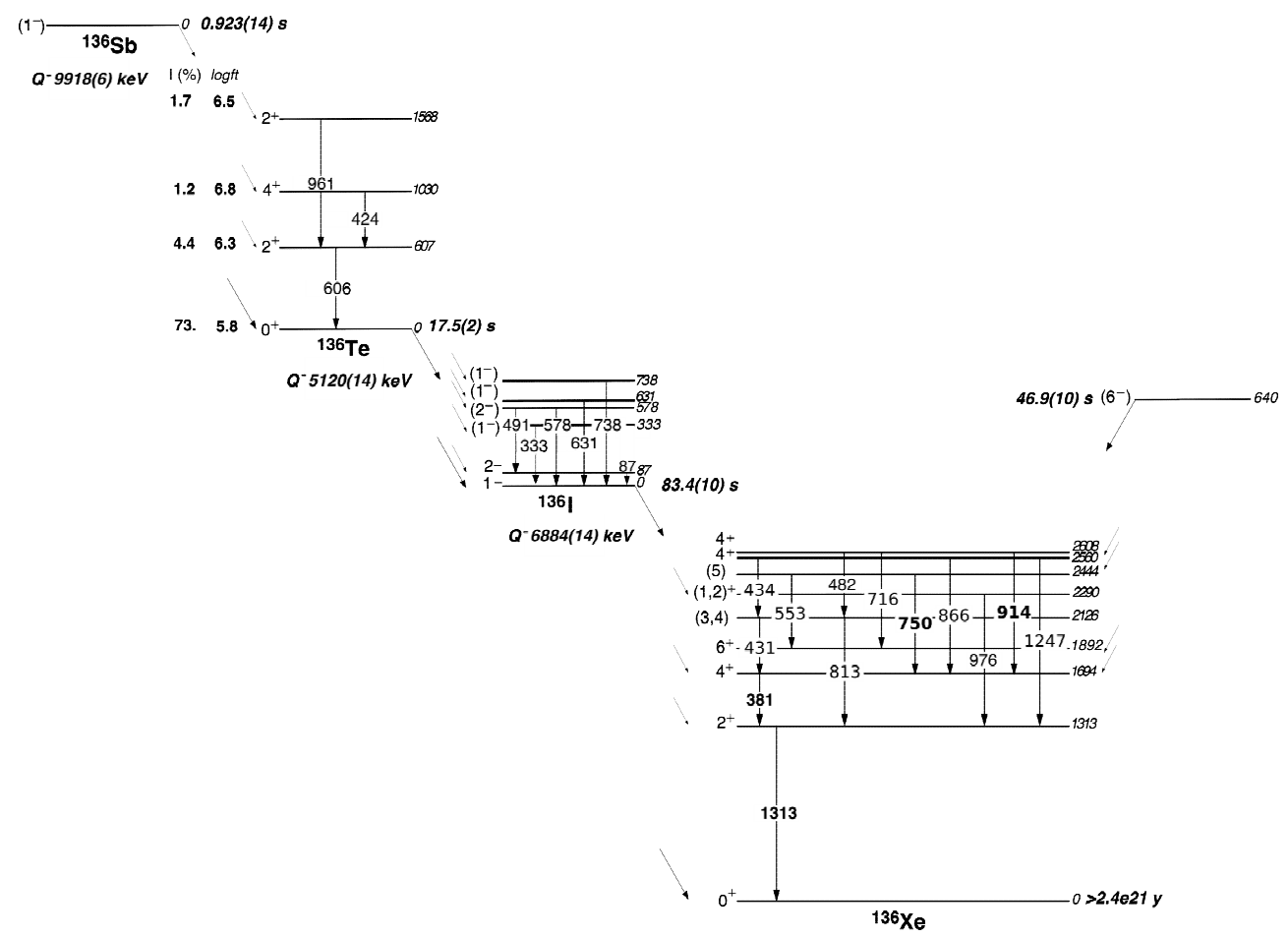

Fig. 3. Decay schemes observed in the experiment, spin/parities are adopted from [28], Q values from [30]. The strong g.s. to g.s. feeding between ${ }^{136} \mathrm{Sb}$ and ${ }^{136} \mathrm{Te}$ is based on the deduced intensities (see text). New information for ${ }^{136} \mathrm{Te}$ is in bold, the (6-) decay transitions in ${ }^{136} \mathrm{Xe}$ are in bold.

\section{Discussion}

The low intensities of transitions following the ${ }^{136} \mathrm{Sb} \rightarrow{ }^{136} \mathrm{Te}$ decay observed in our study support the fact that most of the decay proceeds through the g.s. of ${ }^{136} \mathrm{Te}$, which is in agreement with the earlier conclusions of Hoff et al. [16]. This results in the most probable spin/parity assignment of $\left(1^{-}\right)$for the g.s. of ${ }^{136} \mathrm{Sb}$. Our deduced logft values (for ${ }^{136} \mathrm{Te}$ in Fig. 3) using [31] support this conclusion. For example, assuming the (2-) spin/parity proposed in [17] for the g.s. of ${ }^{136} \mathrm{Sb}$, would increase the feeding to the first and second $2^{+}$ states in ${ }^{136} \mathrm{Te}$, with similar arguments for the weakly fed $4^{+}$state. In addition, we have not observed other strong branches beyond $1.6 \mathrm{MeV}$ excitation energy, also in agreement with [16]. Taking into account the logft values, one can conclude that the $\beta$-decay proceeds by 
transitions of first forbidden type, the strong transition that is preferred in the $\mathrm{Sb}$ case $\pi g_{7 / 2} v f_{7 / 2}{ }^{3} \rightarrow \pi g_{7 / 2}^{2} v f_{7 / 2}^{2}$ was indeed expected in [16,29,18,20,32]. The (2) alternative would imply an exceptionally fast logft of 8.2 first forbidden unique transition [16] to g.s. of ${ }^{136} \mathrm{Te}$, and has not been observed here. The suggested mixture between $\pi g_{7 / 2} v f_{7 / 2}{ }^{3}$ and $\pi h_{9 / 2} v g_{9 / 2}$ $[17,19]$ for the g.s. of ${ }^{136} \mathrm{Sb}$ could also be rejected as both orbitals manifest at higherexcitation energy $[8,9,15,20]$. Any strong configuration mixings will affect the states in ${ }^{136} \mathrm{Te}$ by pushing them e.g. towards lower energies in comparison to the corresponding twoproton and two-neutron systems. The total number of states below $2.5 \mathrm{MeV}$ should not be appreciably different from the total number found in these systems which is not seen here and in [16].

One can note that the g.s. spin/parity of the neighbouring ${ }^{134} \mathrm{Sb}$ was identified as $\left(0^{-}\right)$with a $\left(1^{-}\right)$state close-lying in energy [33]. This was also debated by Sarkar et al. in [17], where both states were proposed as yrast traps based on shell-model calculations using both empirical and realistic interactions. The agreement found for the $\left(0^{-}\right)$assignment was possible using the realistic interaction [19]. For the g.s. of ${ }^{136} \mathrm{Sb}$, except fitting to the assignments in the analog $\mathrm{Pb}$-chain [8] for ${ }^{212} \mathrm{Bi}$ with g.s. spin/parity $\left(1^{-}\right)$[16], a supporting argument for $\left(1^{-}\right)$can be given following its isomeric decay study [9]. The multipolarities of all transitions we have observed following the $\left(6^{-}\right)$sub- $\mu$ s isomer agree indeed better with $\left(1^{-}\right)$assignment, as supported by our shell-model calculations $[9,15]$.

It is interesting to note that the decay of the ${ }^{136} \mathrm{Te} \rightarrow{ }^{136} \mathrm{I}$ also supports strong g.s. to g.s. feeding with spin/parity of $\left(1^{-}\right)$for ${ }^{136} \mathrm{I}$ [28]. New data on this issue will be discussed in a forthcoming article [27].

\section{References}

1. B.A. Brown et al., Phys. Rev. Lett. 85, 5296 (2000)

2. S. Sarkar et al., Phys. Rev. C 81, 064328 (2010)

3. M.-G. Porquet et al., Eur. Phys. J. A 25, 319 (2005)

4. P. Bhattacharyya et al., Eur. Phys. J. A 3, 109 (1998)

5. S.H. Liu et al., Phys. Rev. C 81, 014316 (2010)

6. R. Kshetri et al., Phys. Rev. C 74, 034314 (2006)

7. T. Rzaca-Urban et al., Eur. Phys. J. A 32, 5 (2007)

8. L. Coraggio et al., Phys. Rev. C 80, 021305(R) 2009

9. R. Lozeva et al., Phys. Rev. C 92, 024304 (2015) $\square$

10. H. Wang et al., Prog. Theor. Exp. Phys. 2014, 023 D02 (2014)

11. J. Terasaki et al., Phys.Rev. C 66, 054313 (2002) $\square$

12. D. Bianco et al., Phys. Rev. C 88, 024303 (2013)

13. A. Korgul et al., Phys. Rev. C 64, 021302(R) (2001)

14. J. Shergur et al., Phys. Rev. C 72, 024305 (2005)

15. R. Lozeva et al., Phys. Rev. C 93, 014316 (2016) $\square$

16. P. Hoff et al., Phys. Rev. C 56, 2865 (1997) $\square$

17. S. Sarkar et al., Pramana 53, 469 (1999)

18. P. Lee et al., Phys. Rev. C 92, 044320 (2015) $\square$

19. S. Sarkar et al., Eur. Phys. J. A 21, 61 (2004) $\square$ 
20. B. Moon et al., Phys. Rev. C 95, 044322 (2017)

21. S. Nishimura et al., Prog. Theor. Exp. Phys. 2012, 03 C006 (2012)

22. P.-A. Söderström et al., Nucl. Instrum. Meth. B 317, 649 (2013)

23. P. Mutti et al., ANIMMA (2013), doi:10.1109/animma.2013.6728050

24. J-M. Regis et al., Nucl. Instr. Meth. A 763, 210 (2014)

25. J. Kurpeta et al., Phys. Rev. C 85, 027302 (2012)

26. L. Mathieu et al., JINST 7, P08029 (2012)

27. R. Lozeva et al. (to be published)

28. www.nndc.bnl.gov

29. W.R. Western et al., Phys. Rev. C 15, 1822 (1977)

30. G. Audi et al., Chin. Phys. C 41, 030001 (2017)

31. N.B. Gove, M.J. Martin, Atom. Data Nucl. Data A 10, 205 (1971)

32. I. Borzov, Phys. Rev. C 67, 025802 (2003)

33. B. Fogelberg et al., Phys. Rev. C 4151890 (1990) 